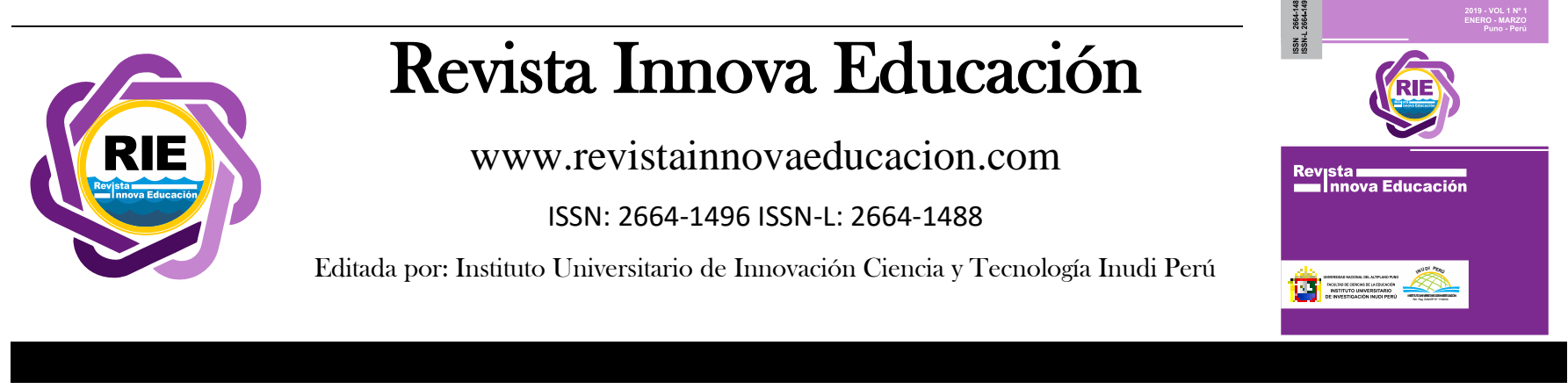

\title{
Aportes a la consolidación del conectivismo como enfoque pedagógico para el desarrollo de procesos de aprendizaje
}

\author{
Contributions to the consolidation of connectivism as a pedagogical approach to the \\ development of learning processes
}

Eileen Bernal-Garzón ${ }^{1}$

Universidad Distrital Francisco José de Caldas, José de Caldas-Bogotá, Colombia

https://orcid.org/0000-0003-4061-3840

DOI: https://doi.org/10.35622/j.rie.2020.03.002

Recibido 28/02/2020/ Aceptado 16/04/2020 Aceptado 26/07/2020

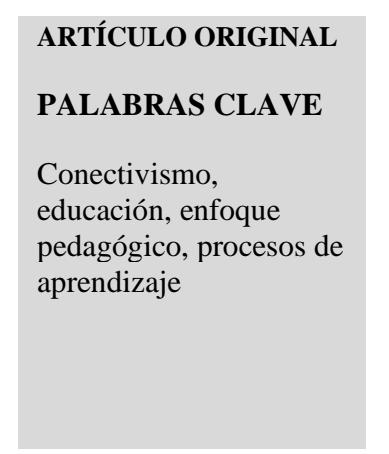

\section{KEYWORDS}

Connectivism, education, pedagogical approach, learning processes

\begin{abstract}
Este artículo reflexiona sobre el conectivismo como un enfoque pedagógico para el desarrollo de procesos de aprendizaje que se pueden generar desde las tecnologías de la información y la comunicación aplicadas a la educación. Se presenta como una alternativa pedagógica que ha pasado por una serie de debates epistemológicos acerca de su carácter teórico y valor pedagógico para transformar las prácticas educativas tradicionales. La metodología que se utilizó fue la revisión documental y recoge las principales investigaciones acerca del conectivismo que se han realizado en países de Latinoamérica, de Estados Unidos y de españa entre otros, y que son referenciadas en diversas bases de datos, como Redalyc, Dialnet, Springer y Scopus. En los resultados se presentan las diferentes discusiones teóricas y conceptuales acerca del conectivismo haciendo énfasis en los aportes que ha tenido para la consolidación de nuevas corrientes pedagógicas y educativas, para el desarrollo de procesos de aprendizaje.
\end{abstract}

${ }^{1}$ Correspondencia: eibernalg@correo.udistrital.edu.co 


\section{INTRODUCCIÓN}

En 2004, George Siemens publica un documento títulado "Conectivismo: Una teoría de aprendizaje para la era digital", en éste expone un análisis acerca de diferentes enfoques de aprendizaje que han rodeado el desarrollo de los ambientes instruccionales y, en otras palabras, de la educación. Dicho autor parte de la idea de que el aprendizaje debe ser personal, y al estar siendo impactado por las tecnologías debe crear en el individuo la "capacidad de sintetizar y reconocer conexiones y patrones para aprender" (Siemens, 2004, p. 5). En este sentido, se puede decir que en medio del auge de las nuevas tecnologías, el individuo está inmerso en un caos de conexiones y patrones, sobre los cuales deberá tomar decisiones y dichas decisiones se convertirán en la base de su conocimiento.

Por su parte, Downes en el 2005 hace referencia a que hay nuevas formas de conocimiento que son creadas por medio de entidades conectadas que tienen interacciones y tienen distribuido el conocimiento. En otras palabras, Downes (2005) afirma que el conocimiento ya no es sólo cuantitativo, ni cualitativo, sino que es una nueva forma de representación e interpretación de las sensaciones que recibimos del mundo en relación con la distribución del conocimiento. Con lo cual se reitera la idea de que el conocimiento no deviene exclusivamente del individuo en sí mismo, sino que éste es y está conectado por entidades que se encuentran en caos y que conforman nuevos patrones que deben ser comprendidos por dicho individuo, a medida que su percepción en relación con el mundo cambia.

\section{a. El conectivismo como enfoque pedagógico}

Ahora bien, siguiendo a Siemens (2004) y a Downes (2006), para comprender mejor el conectivismo es necesario tomar como referente diferentes conceptos que dan lugar a su pertinencia como enfoque pedagógico, ya que a partir de la comprensión de dichos conceptos es posible identificar las discusiones epistemológicas que han surgido en torno al mismo y los aportes de éste para mejorar el desarrollo de procesos de aprendizaje.

\section{$>$ Red}

En palabras de Siemens (2004), "Una red puede ser definida simplemente como conexiones entre entidades. Las redes de computadores, las mallas de energía eléctrica y las redes sociales funcionan sobre el sencillo principio que las personas, grupos, sistemas, nodos y entidades pueden ser 
conectados para crear un todo integrado" (p. 6), sin ir más allá de la definición en sí misma, la red para Siemens (2004), está hecha de conexiones y los puntos que unen esas conexiones se denominan nodos (entidades). No obstante, para Downes (2006), lo que se puede entender por red, debe ser inferido de lo que el define como conocimiento conectivo en el cual existen unas entidades que tienen el conocimiento distribuido, y entre las entidades existen interacciones (conexiones), que dependen de la interpretación producida por fenómenos emergentes (Ver figura No. 1). De acuerdo con lo anterior el conectivismo establece que no hay una red definida que limita el fluir de la información entre los nodos y/o las entidades.

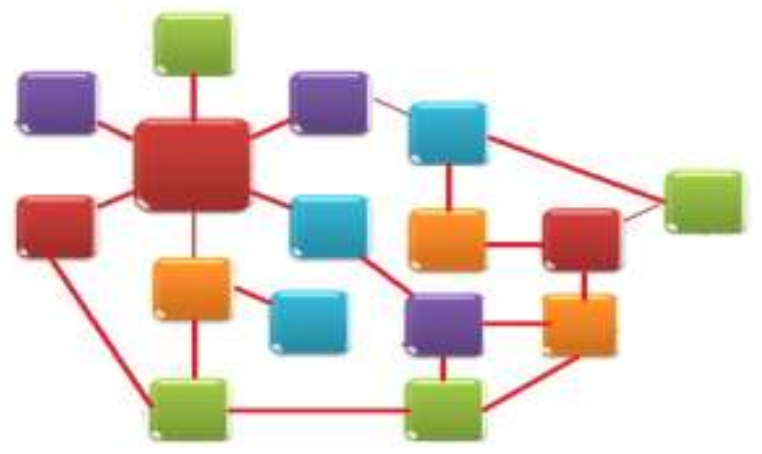

Figura 1 Red, conexiones y entidades (Fuente: Elaboración propia)

\section{$>$ Nodos}

Por otra parte, Siemens (2004) define los nodos desde la teoría de la ciencia de redes, explicando que estos "compiten siempre por conexiones, porque los enlaces representan supervivencia en un mundo interconectado" (Barabasi, 2002, p.106). Para aclararlo aún más Barabasi (2013) plantea que los nodos existen antes que las redes y que una vez haya un enlace que conecte el nodo a la red ésta lo integrará. En este sentido, Downes (2006) toma el mismo concepto de nodo y de los enlaces de éste.

En términos prácticos, los nodos se pueden considerar las comunidades de aprendizaje, que se constituyen de los individuos que participan en la comunidad y de la información con la que cuentan; por otra parte, estos nodos pueden variar en su fuerza para impactar la red dependiendo de la cantidad de individuos que participan y de la cantidad de información con la que cuentan. Y en el caso de que un nodo se quiera integrar a la red, la misma red hará posible su conexión, o sí por el contrario un nodo se aisla de la red, igual conformará una red más pequeña, pero de la misma forma seguirá constituyendo una red. A continuación un gráfico que ejemplifica esta opción. 


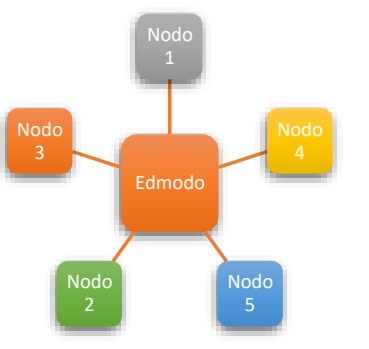

Figura 2 Construcción de una red

(Fuente: Elaboració propia)

Aunque la anterior es una red sencilla, se pueden evidenciar cinco nodos que la configuran, los cuales giran en torno a un nodo mayor que en este caso se puede ejemplificar en la web, con plataformas como Edmodo, la cual constituye una red social educativa, que ofrece a docentes, estudiantes, padres de familia y comunidades educativas en general, la opción de conectar a sus miembros entre sí y con miembros de otras comunidades de aprendizaje, para compartir información, estrategias, actividades, evaluaciones y experiencias de aprendizaje. lo cual se constituirá en una forma de conocimiento conectivo, si tenemos en cuenta que éste tiene como principio el reconocimiento de patrones que le permita a los individuos, en este caso de las comunidades educativas, tener cierta configuración de lo que saben (Leal, 2012).

\section{Auto-organización}

En cuanto al concepto de auto-organización, Siemens (2004) aclara que su teoría del conectivismo parte de "la integración de principios explorados por las teorías de caos, redes, complejidad y auto-organización” (p. 6). De tal forma que articula la concepción del aprendizaje a conceptos como el de red y el de nodo; pero como se evidenció en relación con los conceptos anteriores, la red y los nodos actuan de forma interconectada y como una red puede contar con un sin número de nodos, éstos se conectan por competencia, así que es necesario hablar de autoorganización

El concepto de auto-organización Siemens (2004), lo adopta de Rocha (1998), en donde se dice que "la auto-organización es la formación espontánea de estructuras, patrones o comportamientos bien organizados, a partir de condiciones iniciales aleatorias” (p. 3). Sin embargo, en el caso de Downes (2005), el concepto de auto-organización devendría en la posibilidad de entender ¿qué significa saber? Pues es éste la representación por excelencia del aprendizaje, ya que "está basado en la organización y la conectividad en el cerebro" (Downes, 2005, p. 21) lo que en consecuencia implica que la percepción de Downes (2005), sobre el aprendizaje no es algo estático, sino que depende de la manera en que surgen entornos de 
aprendizaje que ahora están situados en la red y que se exploran por el conocimiento conectivo, que trasciende lo cualitativo y lo cuantitativo.

\section{Aprendizaje}

Finalmente, en cuanto al concepto de aprendizaje se podría decir que a este punto se entra en lo más algido y más esquivo del conectivismo como enfoque de aprendizaje, ya que Siemens (2004), hace referencia al aprendizaje, como "un proceso que ocurre al interior de ambientes difusos de elementos centrales cambiantes - que no están por completo bajo control del individuo" (p. 6). Además explica que dicho aprendizaje "está enfocado en conectar conjuntos de información especializada, y las conexiones que nos permiten aprender más tienen mayor importancia que nuestro estado actual de conocimiento" (Siemens, 2004, p. 6), con lo cual se involucra al individuo como parte de una red, en la cual debe tomar decisiones y dichas decisiones se convertirán en la base de una competencia necesaria para la era digital, en la cual el acceso a la información es casi que ilimitado.

Por su parte, Downes (2005) no habla como tal de aprendizaje, sino que hace una descripción clara sobre la manera en que se da o se crea el conocimiento. Lo cual, se podría decir difiere de una concepción de aprendizaje, pero al mismo tiempo le aporta al conectivismo, teniendo en cuenta que él hace referencia a que el conocimiento es conectivo y depende de la intepretación de un fenomeno emergente (señales) que involucran diferentes entidades (nodos), que interactuan (se conectan) desde patrones de interpretación establecidos por cualidades físicas que se infieren y se asocian por medio de conexiones con lo ya conocido, creando así redes.

$\mathrm{Y}$ es en esta diferente concepcion sobre el aprendizaje entre Siemens y Downes que han surgido una serie de variables para comprender los aportes del conectivismo en el ámbito pedagógico y educativo tema del cual deviene este documento de reflexión.

\section{MÉTODO}

El artículo de reflexión tuvo como propósito analizar los aportes a la consolidación del conectivismo como enfoque pedagógico para el desarrollo de procesos de aprendizaje, partiendo de la teoría de Siemens (2004) y Downes (2006), por tal razón el artículo recopila diferentes concepciones pedagógicas en las que se ha insertado el conectivismo desde el 2004. El documento, recoge un total de 50 referencias bibliográficas, entre artículos de investigación y capítulos de libros, que abordan los principales debates epistemológicos del conectivismo:

$\checkmark$ El conectivismo como una visión pedagógica 
$\checkmark$ El conectivismo como marco teórico para comprender el aprendizaje

$\checkmark$ El conectivismo, aprendizaje en red y redes de aprendizaje

$\checkmark$ El conectivismo y los entornos personales de aprendizaje

$\checkmark$ El conectivismo como fundamento para el diseño de modelos instruccionales de aprendizaje

Con el fin de presentar un amplio espectro de la consolidación del conectivismo en el ámbito pedagógico presentando sus bases teóricas y los ejes pedagógicos que los definen, la metodología que se utilizó fue la revisión documental y recoge las principales investigaciones acerca del conectivismo que se han realizado en países de latinoamérica, de Estados Unidos de españa, entre otros, y que son referenciadas en diversas bases de datos, como Redalyc, Dialnet, Springer, Scopus y google académico. La revisión documental se realizó por medio de acceso a dichas bases de datos, ya qué estas manejan temáticas en torno al tema de la inmersión de las tecnología de la información y la comunicación en la educación.

\section{RESULTADOS Y DISCUSIONES}

a. El conectivismo como una visión pedagógica

Varios autores, desde el 2008, Kop \& Hill (2008), Ravenscroft (2011), Şahin \& Abu Safieh (2012), Adell (2013), Morras (2014), Steffens, et al (2015), Bair \& Stafford (2016) entre otros, han destacado el trabajo de Verhagen (2006), como una de las principales alternativas a la comprensión del conectivismo, ya que éste indica que el conectivismo como tal no es una teoría de aprendizaje, sino que se trata de una visión pedagógica que puede ser ajustada a los planes de estudio pues éstos se ocupan del ¿qué se aprende? y ¿por qué?

Así los principios de aprendizaje que postula Siemens (2004), de acuerdo con Verhagen (2006), pueden ser agrupados en cuatro bases para el desarrollo de un plan de estudio que atienda a las exigencias de aprendizaje de la era digital.

$\checkmark$ Se debe promover la capacidad de ver conexiones entre campos, ideas y conceptos.

$\checkmark$ Se deben nutrir y mantener conexiones necesarias para facilitar el aprendizaje continuo.

$\checkmark$ Se debe promover la habilidad de elegir qué aprender y el significado de la información que se ve a través de la lente de una realidad cambiante.

$\checkmark$ El aprendizaje puede residir en aparatos no humanos.

De lo contrario, los principios de Siemens (2004), no pasan de ser postulados que no pueden ser verificables en la práctica, lo cual le quita el carácter de teoría de aprendizaje al conectivismo. 
Incluso Duke, B., Harper, G., \& Johnston (2013), afirman que "Si el conectivismo se considera una teoría del aprendizaje en lugar de una teoría de la simple conexión, debería haber una transferencia y promoción de la comprensión del alumno" (p. 8), con lo que se puede evidenciar que la crítica de Vergahen (2006) al conectivismo, ha sido estudiada y revisada, a la luz de sus posibilidades para determinar el rol del estudiante en el aprendizaje.

En lo que se refiere al tema del aprendizaje, Verhagen (2006), explica que Siemens (2004), lo entiende a éste como un resultado más no como un proceso, y que por eso afirma que el aprendizaje puede residir en dispositivos no humanos, lo cual implica que la teoría de Siemens no diferencia entre el aprendizaje de personas y los objetos de aprendizaje; esto resulta cuestionable en términos de la comprensión de las actividades de aprendizaje humano, que tienen que ver con el razonamiento y el entendimiento. En otras palabras, Verhagen (2006) afirma que los postulados de Siemens, se pueden ajustar a temas relacionados con el aprendizaje de las máquinas programadas algorítmicamente, pero en ningún caso esto tiene que ver con el modo en que se da el aprendizaje humano.

La crítica de Vergahen (2006) se puede considerar una de las más claras en relación con el modo en que se toma el conectivismo como una teoría de aprendizaje; ya que, Siemens (2004), al basarse en la teoría del caos, en la ciencia de redes y en los parámetros de la auto-organización. Está haciendo referencia al aprendizaje como algo dado y que, al residir en los dispositivos no humanos, como tal sería algo terminado. Cabe aclarar en este caso que la crítica de Verhagen (2006), en medio de ser válida no está tomando en cuenta los postulados de Downes (2005), sobre el conocimiento conectivo, el cual podría resolver esta crisis del conectivismo en torno a lo que se concibe como aprendizaje.

Ahora bien, la crítica de Verhagen (2006), se ha tomado como una base para hablar del conectivismo desde otros contextos de aprendizaje, que incluyen el tema de la pedagogía como una parte esencial para mejorar los procesos educativos; lo cual ha dado paso a nuevas formas de entender el conectivismo. Por ejemplo, Morras (2014), cita que "Algunos autores (Verhagen, 2006; Kop y Hill, 2008; Bell, 2011) dudamos de que el conectivismo pueda ser considerado como una teoría del aprendizaje; en todo caso constituiría una propuesta pedagógica acorde con las nuevas realidades derivadas de la web 2.0 (p. 40)".

\section{b. El conectivismo como marco teórico para comprender el aprendizaje}

Kop \& Hill (2008) agregan una variable diferente a la crítica al conectivismo que había iniciado con Verhagen (2006), ya que plantean la pregunta de sí ¿el conectivismo es una nueva teoría de 
aprendizaje para el futuro o por el contrario es un vestigio de las teorías de aprendizaje del pasado? o sí por otra parte ¿el conectivismo puede erigirse como un marco teórico para comprender el aprendizaje en la era del siglo XXI?

Para responder a esto, Kop \& Hill (2008), parten de la definición del concepto de teoría, estableciendo que ésta debe cumplir con unos criterios específicos que le permitan constituirse como tal. En este sentido, una teoría "debe estar dentro del dominio de Investigaciones científicas, utilizar métodos científicos y basarse en estudios. Debe ser lógicamente construida y verificable a través de pruebas".

$\mathrm{Al}$ respecto, es de aclarar que el conectivismo si parte de investigaciones científicas, pero que no están relacionadas con el campo de investigación del aprendizaje, sino que están enmarcadas en ciencias diferentes al mismo, como la ciencia de redes y la teoría del caos. Cuando la teoría fue planteada en el 2004, por Siemens, no contaba con pruebas y/o investigaciones aplicadas que permitieran determinar la veracidad de sus postulados, pues el conectivismo se fundamenta en las experiencias de cursos Mooc, los cuales de acuerdo con Downes (2013) empiezan en 2008. Por tanto se puede deducir que el conectivismo no cumple con la condición de ser una teoría.

En vista de esta carencia del conectivismo, Kop \& Hill (2008) destacan que existen las teorías del desarrollo, las cuales pueden considerarse como teorías emergentes y que éstas "pueden conducir a una investigación empírica que valida o refuta las hipótesis formales postuladas en el marco del método científico". Con lo cual se podría comprender al conectivismo, no como teoría del aprendizaje, sino como una teoría emergente del aprendizaje. Al respecto, un elemento clave que reitera esta idea, es que sólo cuatro años después de planteada la teoría, Siemens (2008) va a empezar a integrar categorías de comprensión del aprendizaje basadas en los principios teóricos del objetivismo, del pragmatismo y de la interpretación; las cuales le permitieron al conectivismo ofrecer una base conceptual diferente y alternativa del aprendizaje, resaltando que éste parte de una realidad que es externa a la mente y que existe una negociación entre la reflexión, la experiencia y la investigación que se dan por medio de la socialización y de las señales generadas por conocimiento distribuido.

c. El conectivismo, aprendizaje en red y redes de aprendizaje El aprendizaje en red es un fenómeno que se empieza a consolidar como parte esencial de la manera en cómo aprenden los individuos en relación con la aparición de las nuevas tecnologías de la información y la comunicación en el siglo XXI, y aunque existen antecedentes que pueden 
hablar del aprendizaje en red a nivel pedagógico, se podría decir que éste adquiere todo su peso epistemológico y conceptual en relación con el conectivisimo como un marco teórico (Kop \& Hill, 2008), que permite definir los principios de acción de dicho aprendizaje en red.

En este sentido van a aparecer estudios como el de Duke, et al, (2013), que van a hablar sobre comunidades de aprendizaje con formas de socialización conectivistas; éste estudio resalta que el conectivismo:

"se caracteriza como un reflejo de nuestra sociedad que está cambiando rápidamente a una sociedad que es más compleja, conectada social y globalmente y mediada por el aumento de los avances en tecnología, por lo que el aprendizaje conlleva la orquestación de una desorganización compleja de ideas, conectada en red para formar conjuntos de información" (Duke, et al, 2013, p. 6).

Por lo cual se podría establecer que el conectivismo constituye la base teórica que da lugar a la comprensión del modo en que se da el aprendizaje en red; ya que éste precisa el modo en que opera y reacciona la sociedad y la escuela ante las transfomaciones producto de la tecnología, y por lo mismo contribuye a la conformación de directrices que orienten la comprensión de los cambios en el aprendizaje, como parte estructural de ésta sociedad. De acuerdo con Duke, et al, (2013) "el conectivismo es sin duda, una importante escuela de pensamiento directamente aplicable a la utilización de la tecnología en el aula en la actualidad". Y éste ofrece una "configuración de redes que pueden ayudar a las nuevas generaciones a colaborar para encontrar soluciones a un número cada vez mayor de preguntas" (p. 9).

$\mathrm{Al}$ respecto, los estudios sobre el aprendizaje en red y las redes de aprendizaje, comparten con el conectivismo conceptos claves como el de sociedad de la información, sociedad del conocimiento, era digital y competencias para el siglo XXI. Además citan entre otros, el trabajo de Siemens (2004, 2008, 2010), como un referente que da lugar al concepto de aprendizaje en red, ubicándolo como una apuesta para la reformulación del sistema educativo y el fomento de competencias a lo largo de toda la vida, tanto para niños, como para adolescentes, y para profesionales (Sloep \& Berlanga, 2011).

Por otra parte, el estudio de Bartolome (2011) es uno de los más representativos que hace referencia directa a la correspondencia teórica entre el conectivismo, el aprendizaje en red y redes de aprendizaje, ya que, se refiere al aprendizaje como "un proceso de conectar y generar información en el contexto de una comunidad de aprendizaje” (Bartolomé, 2011, p. 71), resaltando que no puede haber sólo una forma de entender cómo se da o se genera el aprendizaje, sino que 
evidentemente, cualquier intento por comprender el aprendizaje debe responder a los diferentes enfoques que han estudiado éste, como el constructivismo y el cognitivismo, retomando así los postulados de Kop \& Hill (2008).

Bartolomé (2011) señala que aprender desde una perspectiva conectivista implica incrementar el conocimiento de un individuo. En otras palabras, "si hemos aceptado que el conocimiento no reside en el individuo ni tan solo en el grupo, sino en las interacciones en el grupo, habrá que aceptar que aprender no es el enriquecimiento del individuo sino el proceso por el que se incrementan esas interacciones" (Bartolomé, 2011, p. 73), y esto es lo que se ha dado en conocer como aprendizaje en red. La particularidad en relación con el conectivismo es que la red, no sólo es el espacio o el medio en el que se produce el aprendizaje sino que también es un ente al que se revierte el aprendizaje. Desde otra perspectiva, Steffens, et al (2015) resalta que, el conectivismo habla de lo que implica el aprendizaje en red, en tanto la aplicación de la teoría de Siemens (2004) y de Downes (2005) se vio reflejada en la creación y diseño de cursos masivos abiertos y en línea (MOOCs), que tenían el propósito de ofrecer un marco de referencia del modo en que opera el conectivismo

Para el caso de los estudios sobre redes de aprendizaje se puede resaltar que éstos se caracterizan por presentar a las redes de aprendizaje como "entornos de aprendizaje en línea que ayudan a los participantes a desarrollar sus competencias colaborando y compartiendo información" (Sloep \& Berlanga, 2011, p. 56), de tal forma que puedan compartir experiencias, trabajar colaborativamente en proyectos, crear grupos de trabajo, ofrecer y recibir apoyo de otros usuarios de la red de aprendizaje y evaluarse como comunidades de aprendizaje con unos propósitos específicos orientados a la mejora y el cambio educativo. Murillo (2009) cita que las redes de aprendizaje se pueden agrupar en función de su foco de transformación educativa en:

$\checkmark$ Redes que buscan la innovación en el aula a través de la aplicación de experiencias compartidas de docentes que tienen intereses disciplinares y académicos similares.

$\checkmark$ Redes que buscan mejorar las escuelas, por medio de grupos de docentes que comparten la organización y las estructuras comunes de sus escuelas con el fin de generar conocimientos que les permitan transformar sus entornos educativos.

$\checkmark$ Redes que buscan impactar y contribuir a la transformación de la educación en general, desde la investigación y el desarrollo de la innovación educativa. (Murillo, 2009)

En síntesis, desde la perspectiva del conectivismo en relación con el aprendizaje en red según Duke, et al, (2013) se puede decir que "no hay duda de que el aprendizaje en línea es una 
respuesta tecnológica directa para el aprendizaje de diferentes culturas, métodos e inspiraciones"; pues, "cualquiera puede participar y realizar diversas funciones: por ejemplo, estudiantes, profesores, «coaches», mentores, curiosos interesados, individuos que buscan apoyo, etc" (Sloep \& Berlanga, 2011, p. 57), siempre y cuando existan unos propósitos comunes que impliquen trabajar colectivamente con colaboración y compromiso para generar y adquirir conocimiento, desde la negociación y la autonomía.

\section{d. El conectivismo y los entornos personales de aprendizaje}

El concepto de entornos personales de aprendizaje, PLE (Personal Learning Enviroment), surge en el 2004 en el desarrollo del proyecto NIMLE, citado por Adell \& Castañeda (2013), sin embargo es Álvarez (2014), quien especifica que en el marco del proyecto NIMLE, en el cual se desarrolla el congreso JISC (Joint Information Systems Committee), es donde aparece el concepto de PLE, pues en dicho congreso, "se expone la necesidad, y posibilidad, de desarrollar plataformas para la gestión del aprendizaje (LMS) menos centradas en las necesidades de control de las organizaciones y con más posibilidades de personalización para el alumno" (Álvarez, 2014, p. 15). Lo cual nos lleva a establecer la conexión directa que existe entre el conectivismo y los PLE, pues ambos tienen su base en el desarrollo de los LMS, desde un enfoque pedagógico.

En este sentido la importancia de los entornos personales de aprendizaje en relación con el auge del conectivismo, deviene en el hecho de que las fuentes de las que aprenden los individuos han variado y se han consolidado en internet, haciendo que algunos de los elementos educativos de la escuela tradicional, entren en conflicto con los objetivos que deben lograr para promover el aprendizaje. De tal manera, los postulados sobre los entornos personales de aprendizaje siguiendo a Adell, et al, (2013), que se pueden asociar a los principios del conectivismo, parten de las siguientes premisas:

$\checkmark$ Los profesores no son ahora la única fuente de información especializada.

$\checkmark$ La evaluación estándar, deja de lado el aprendizaje personal.

$\checkmark$ La creencia de que el conocimiento reside en la cabeza de las personas, deja de lado el hecho de que el conocimiento depende de recursos externos con los que establecemos relaciones,

$\checkmark$ El conocimiento está en soportes digitales o en red. 
Ahora bien, en Webinar \#4: Entornos personales de aprendizaje ${ }^{2}$, se cita el trabajo realizado por Adell \& Castañeda (2010), en donde se define el entorno personal de aprendizaje, como un "conjunto de herramientas, fuentes de información, conexiones y actividades que cada persona utiliza de forma asidua para aprender".

Por otra parte, Adell (2014) hace referencia a que un elemento vital para un buen desarrollo de un entorno personal de aprendizaje a través de la red, debe tener como principio, la selección de la información, bajo el hecho de que, "el conocimiento no se "localiza", no es acumulable, de que nuestro mayor capital cognitivo no es lo que "tenemos en la cabeza", sino las redes de conexiones entre informaciones y las relaciones que forman esas conexiones. Esas redes y conexiones se organizan y evidencian gracias a nuestro PLE." (Adell, et al, 2013, p. 32).

En otro sentido, Gros (2015) afirma que la escuela ya no es el espacio-centro del saber, sino que se ha convertido en un espacio-nodo del saber, lo que implica qué dicha escuela en la sociedad de la información, ya no es el centro, sino que constituye un nodo más de la red, además que "las tecnologías móviles permiten a los estudiantes de todas las edades operar a través de diferentes contextos.” (Gros, 2015, p. 61). Esta posición acerca aún más los postulados del conectivismo a los principios que fundamentan los entornos personales de aprendizaje, de hecho en términos generales, se puede establecer que, los entornos personales de aprendizaje según Gros (2015) representan el escenario donde se da el aprendizaje de forma conectiva. En consecuencia, un "PLE puede ser visto como una plataforma basada en las redes sociales, centrada en el aprendiz y diseñada para permitir compartir, colaborar y producir recursos y contenidos a través de procesos de participación distribuida.” (Gros, 2015, p. 62).

e. El conectivismo como fundamento para el diseño de modelos instruccionales de aprendizaje

Los modelos instruccionales de aprendizaje, constituyen un escenario nuevo en el que se empiezan a mezclar los postulados del conectivismo, con los principios aplicados del aprendizaje en red, teniendo en cuenta la ubicuidad de las tecnologías digitales. "Es en este último aspecto en el que principalmente se basa el diseño instruccional, que se fundamenta en identificar cuáles son los métodos que deben ser utilizados en el diseño del proceso de instrucción, y también en determinar en qué situaciones estos métodos deben ser usados" (Fernández, et al, 2017, p.1).

\footnotetext{
${ }^{2}$ Video explicativo realizado por Jordi Adell en el marco del proyecto Eduland. (2014) Disponible en: https://www.youtube.com/watch?v=xKUiBD6Ckmg
} 
Por su parte, Zapata-Ros (2012) expone las bases para la construcción de un modelo teórico de aprendizaje y de la elaboración del conocimiento a partir de entornos conectados de aprendizaje. Zapata-Ros (2012), parte de que el aprendizaje "es el proceso o conjunto de procesos a través del cual o de los cuales, se adquieren o se modifican ideas, habilidades, destrezas, conductas o valores, como resultado o con el concurso del estudio, la experiencia, la instrucción, el razonamiento o la observación" (p. 5), esto conlleva a que el conocimiento tenga significado, valor, sea operativo en contextos diferentes y pueda ser representado y transmitido a otros individuos.

Ahora bien, Bair, R. \& Stafford, T. (2016) presentan la discusión entre la teoría del diseño multimedia y el conectivismo, estableciendo que la web 2.0 ha cambiado drásticamente las posibilidades de los diseñadores para crear modelos de instrucción de aprendizaje, debido a que la web 2.0 "ha permitido a los estudiantes, localizar, ensamblar, modificar y transferir documentos y otros archivos en todo el mundo" (Bair, et al, 2016, p. 129).

Desde esta perspectiva el conectivismo, puede representar una salida a los nuevos modelos, ya que, dicho conectivismo entendido como teoría "conducirá a la consideración de la amalgama de tecnologías de e-learning y de aprendizaje móvil y, por consiguiente, podrá forjar un camino hacia un formato más omnipresente que permita mayor autonomía a los instructores" (Bair, et al, 2016, p. 130). Tal como lo realizó Downes (2008), a través de sus cursos masivos abiertos en línea (MOOC), según cita Morras (2014), destacando que "en paralelo a esta nueva teoría del aprendizaje del conectivismo surgen nuevos modelos instructivos como, por ejemplo, el concepto de e-learning 2.0 acuñado por Stephen Downes para simbolizar la aplicación de las herramientas de la Web 2.0 en la educación" (p. 40).

De acuerdo con lo expuesto sobre el conectivismo, en la primera década del siglo XXI se podría considerar que éste pasó de ser identificado como una teoría para el aprendizaje, a un marco de referencia para entender el aprendizaje. Y en lo que va de la segunda década del siglo XXI ha pasado a entenderse como un modelo de aprendizaje en red y/o como un método para diseñar modelos instruccionales de aprendizaje, entre otras interpretaciones.

Molina (2016) en este sentido resalta que:

"Al interior del modelo pedagógico conectivista, se alberga un tipo de aprendizaje que se ha denominado de múltiples formas: aprendizaje ubicuo, aprendizaje invisible, aprendizaje situado, aprendizaje interactivo, aprendizaje digital, entre otros. Todas estas propuestas de aprendizaje tienen un elemento común: la ubicuidad de las Tecnologías de información y 
comunicación Tic, en nuestra vida cotidiana y particularmente en los procesos educativos. $\left(\right.$ Molina, 2016) ${ }^{3}$

Es así que el conectivismo visto como teoría de aprendizaje emergente o como pedagogía emergente, proporciona un enfoque de interpretación del aprendizaje distinta al planteado por las pedagogías tradicionales, en donde el estudiante resultaba ser un receptáculo de información obtenida por repetición. Ahora se habla de aprendizaje por descubrimiento, de aprendizaje ubicuo, de aprendizaje significativo, etc. Así como de ambientes y de ecologías de aprendizaje que favorecen habilidades en los estudiantes del siglo XXI.

\section{CONCLUSIONES}

El conectivismo planteado por Siemens desde su aparición en 2004 como teoría pedagógica ha hecho aportes significativos al desarrollo de nuevas concepciones sobre el aprendizaje y el modo en que éste debe ser entendido en pleno auge del siglo XXI, en tanto es posible evidenciar que los cambios y los retos que está afrontando la educación requieren con urgencia de nuevas perspectivas pedagógicas y educativas que faciliten la articulación entre las tecnología de la información y la comunicación y la educación.

El conectivismo se puede concebir como un proceso de conexión de información que independientemente de su fuente o del nodo que la provee, tiene la posibilidad de generar diferentes tipos de aprendizaje, que pueden ser aplicados dentro y fuera de las aulas de clase, lo cual lleva a su consolidación como estrategia pedagógica para mejorar procesos de aprendizaje y esto se refleja en la amplia transición teórica que ha tenido durante la primera y segunda década del siglo XXI.

El conectivismo entendido como un enfoque pedagógico para comprender el aprendizaje ha generado un debate muy enriquecedor a nivel educativo que le ha permitido trascender la teoría e insertarse en las aulas de clase como modelo instruccional de aprendizaje, deslocalizando así las pedagogías tradicionales para ofrecer nuevos modelos de aprendizaje, como los PLE, que hoy en día aportan a la consolidación de estrategias de articulación de las tic en la escuela tradicional, ofreciendo una amplia gama de posibilidades de aprendizaje para los estudiantes, quienes se reconocen como la parte central de dichos procesos de aprendizaje.

\footnotetext{
${ }^{3}$ Documento realizado por Ruth Molina (2019) en el marco del seminario aprendizajes en red de la maestría en educación en tecnología. Universidad Distrital Francisco José de Caldas
} 
El conectivismo como teoría de aprendizaje emergente o como pedagogía emergente, aún tiene retos que afrontar en tanto pueda identificar y desarrollar procesos de adquisición del conocimiento, que puedan ser usados que más allá de ser instruccionales y constituyan una verdadera guía para el impulso y perfeccionamiento de métodos de aprendizaje que les faciliten a los estudiantes "aprender a aprender" a identificar redes, nodos y a auto-organizar su aprendizaje en torno no sólo a intereses personales sino a contenidos propios de los currículos académicos.

\section{REFERENCIAS BIBLIOGRÁFICAS}

Adell, J. (25 de 09 de 2014). Webinar \#4: Entornos personales de aprendizaje. Recuperado el 08 de 06 de 2017, de Eduland.es: https://www.youtube.com/watch?v=xKUiBD6Ckmg

Adell, J. (2012). Tecnologías emergentes, ¿pedagogías emergentes? En J. P. Hernández, Tendencias emergentes en educación con TIC (págs. 13 - 32). España: Ediciones Espiral, educación y tecnología.

Adell, J. (2013). El ecosistema pedagógico de los PLEs. En C. Adell, J., \& Editorial Marfil S.A. (Ed.), Entornos Personales de Aprendizaje: Claves para el ecosistema educativo en red (págs. 29 - 52).

Altamirano, E., Becerra, N., Nava, A. (2010). Hacia una educación conectivista. Revista Alternativa, 22, 22 - 32.

Álvarez, D. (2014). Una respuesta global ante los nuevos retos: Los entornos personales de aprendizaje. En D. Álvarez, Entornos personales de aprendizaje (PLE): aprendizaje conectado en red (pág. 134). España: Ministerio de educación, cultura y deporte.

Bair, R. \& Stafford, T. (2016). Connected and Ubiquitous: a Discussion of Two Theories That Impact Future Learning Applications. TechTrends, 60(2), 129-135.

Barabasi, A. (2013). Network science. Philosophical Transactions of the Royal Society A: Mathematical, Physical and Engineering Sciences.

Barnett, J., McPherson, V., \& Sandieson, R. (2013). Connected teaching and learning: The uses and implications of connectivism in an online class. Australasian Journal of Educational Technology, 29(5).

Bartolomé, A. (2011). Conectivismo: Aprender en red y en la red. En A. Bartolomé, Teconologias na educação: Uma abordagem crítica para uma atualização prática (págs. 71-86). 
Bartólome, A. (2012). De la web 2.0 al elearning 2.0. Perspectiva. Florianópolis, 30, 131 - 153.

Bell, F. (2011). Connectivism: Its place in Theory-informed research and innovation in technology-enabled learning. International Review of Research in Open and Distance Learning, 12(3).

Del Valle, I. (2009). Teoría de la conectividad como solución emergente a las estrategias de aprendiaje innovadoras. Revista electrónica de humanidades, educación y comunicación social, 6(4), $1-25$.

Domenech, F. (2014). La enseñanza y el aprendizaje en la situación educativa. En F. Domenech, Aprendizaje y desarrollo de la personalidad (págs. 1 - 11).

Downes, S. (22 de Diciembre de 2005). An Introduction to Connective Knowledge. Obtenido de Stephen's Web: www.downes.ca/cgi-bin/page.cgi?post=33034

Downes, S. (2006). Connectivism and Connective Knowledge Essays on meaning and learning networks. (National Research Council Canada, Ed.) Canadá.

Downes, S. (03 de 02 de 2007). Half an hour- A place to write, half an hour, every day, just for me. Recuperado el 05 de 08 de 2017, de What Connectivism Is: http://halfanhour.blogspot.com.co/2007/02/what-connectivism-is.html

Downes, S. (10 de Julio de 2013). La condición semántica: conectivismo y aprendizaje abierto. Video conferencia. Canada: Organización de Estados Iberoaméricanos. Disponible en: https://www.youtube.com/watch?v=Oth_9v3RcuI.

Duart, J. (2011). La Red en los procesos de enseñanza de la Universidad. Comunicar: Revista científica iberoamericana de comunicación y educación, XIX(37), 10-13.

Duke, B., Harper, G. \& Johnston, M. (2013). Connectivism as a Digital Age Learning Theory. En M. Duke, B., Harper, G., \& Johnston, \& \&. Lorraine Stefani, Shoba Tegginmath (Ed.), The International HELT Review (2013 ed., págs. 4 - 13). New York: The International HETL Association .

Elliott, R. \& Shaun, M. (2011). Connectivism's Role as a Learning Theory and its Application in the Classroom. Boise State University, 1 - 9 . 
Fernández, R. (15 de 05 de 2017). Aprendizaje con nuevas tecnologías paradigma emergente. ¿Nuevas modalidades de aprendizaje? Recuperado el 12 de 06 de 2017, de Educrea: https://educrea.cl/aprendizaje-con-nuevas-tecnologias-paradigma-emergente-nuevasmodalidades-de-aprendizaje/

Garcia, E., Elbeltagi, I., Brown, M., \& Dungay, K. (2015). The implications of a connectivist learning blog model and the changing role of teaching and learning. British Journal of Educational Technology, 46(4).

Gros, B. (2015). La caída de los muros del conocimiento en la sociedad digital y las pedagogías emergentes. Teoria de la Educacion.

Gutiérrez, L. (2012). Conectivismo como teoría de aprendizaje: conceptos, ideas y posibles limitaciones. Revista educación y tecnología. Págs. 111-122, 1(1), 111-122.

Kop, R. \&. Hill, A. (2008). Connectivism: Learning theory of the future or vestige of the past? The international review of research in open and distance learning. 9 (3). Obtenido de: http://www.irrodl.org/index.php/irrodl/article/view/523/1103

Leal, D. (8 de Junio de 2012). Conectivismo: el reto de pensar y aprender en red. Video conferencia. Organización de Estados Iberoaméricanos. Disponible en: https://www.youtube.com/watch?v=5Kpoo2vZkeQ.

Ledesma, A. (2015). Del conductismo, cognitivismo y constructivismo al conectivismo. En A. Ledesma, \& Editorial Jurídica del Ecuador (Ed.), Conectivismo (1 ed., págs. 14 - 74). Ecuador.

Martínez, M. (2006). Validez y confiabilidad en la metodología cualitativa. Paradigma, 7 - 33.

Molina, R. (2016). Tipos de aprendizaje en red. En R. Molina, Aprendizajes en red. Bogotá Colombia: Universidad Distrital Francisco José de Caldas.

Morras, A. (2014). Aportaciones del conectivismo como modelo pedagógico post-constructivista. Propuesta Educativa, 2(12), 39 - 48.

Morrás, A. (2011). Proceso de enseñanza-aprendizaje y web 2.0: valoración del conectivismo como teoría de aprendizaje post-constructivista. Estudios sobre educación, 117 - 140. 
Murillo, J. (2009). Las redes de aprendizaje como estrategia de mejora y cambio educativo. Revista Iberoamericana sobre Calidad, Eficacia y Cambio en Educación, 7(3), 3 - 6.

Ordoñez, L. (2015). Cultura participativa y conectivismo: Algunos retos para la investgación latinoamericana. Research Gate(January 2013), 289 - 308.

Ovalles, L. (2014). Conectivismo ¿Un nuevo paradigma en la educación actual? Revista Alternativa, 7, 72 - 79.

Padilla, J., Vega, P., \& Rincón, D. (2014). Tendencias y dificultades para el uso de las TIC en educación superior. Entramado, 10(1), 272-295.

Ramírez, M. (2015). Formación de equipos docentes para facilitar la conexión de la enseñanza en MOOC. Revista interuniversitaria de formación del profesorado, ISSN 0213-8646, $N^{\circ} 83$, 2015 (Ejemplar dedicado a: Enseñanza y aprendizaje en Educación Superior), págs. 2943, 83(83), 29-43.

Ravenscroft, A. (2011). Dialogue and Connectivism: A New Approach to Understanding and Promoting Dialogue-Rich Networked Learning Introduction: Connectivism and Learning in the Digital Age. International Review of Research in Open and Distance Learning, 12.

Reese, S. (2015). Online learning environments in higher education: Connectivism vs. dissociation. Education and Information Technologies, 20(3).

Rittberger, M. \& Blees, I. (2009). Entorno de aprendizaje de la Web 2.0: Concepto, aplicación y evaluación. Elearningpapers, No. 15, 1-20.

Rochefort, B. \& Richmond, N. (2011). Conectar la enseñanza a las tecnologías interconectadas ¿ Por qué es importante? La perspectiva de un diseñador pedagógico. Revista de Universidad y Sociedad del Conocimiento, 8(1), 200-217.

Sánchez, M. (2012). Diseño de recursos digitales para entornos e-Learning en la enseñanza universitaria. RIED. Revista Iberoamericana de Educación a Distancia, Juilo, 53-74.

Siemens, G. (2004). Connectivism: A Learning Theory for the Digital Age. Obtenido de elearnspace everything elearning: http://www.elearnspace.org/Articles/connectivism.htm

Siemens, G., Leal, D. (2007). Conectivismo: Una teoría de aprendizaje para la era digital. En D. Siemens, G., Leal, Conectados en el ciberespacio (págs. 77-90). 
Sloep, P. \& Berlanga, A. (2011). Learning networks, networked learning. Comunicar.

Steffens, C., Bannan, B., Dalgarno, B., Bartolomé, A., Esteve González, V., Cela, J., \& otros. (2015). Recent Developments in TechnologyEnhanced Learning: A Critical Assessment. RUSC. Universities and Knowledge Society Journal, 12(2), 7386.

Tumino, M. \& Bournissen, J. (2016). Conectivismo: Hacia el nuevo paradigma de la enseñanza por competencias. European Scientific Journal, 1212(1010), 112 - 128.

Valerio, G. Valenzuela, J. (2011). Contactos de redes sociales en línea como repositorios de información. Revista de Universidad y Sociedad del Conocimiento, RUSC, 8, 128-155 ST - Contactos de redes sociales en línea.

Valerio, G. Valenzuela, J. (2011). Competencias Informaticas Para el e-learning 2.0. RIED. Revista Iberoamericana de Educación a Distancia, 14(1), 137-160.

Verhagen, P. (2006). Connectivism: A new learning theory? University of Twente. Obtenido de: http://elearning.surf.nl/e-learning/english/3793

Zapata-Ros, M. (2012). Teorías y modelos sobre el aprendizaje en entornos conectados y ubicuos. Bases para un nuevo modelo teórico a partir de una visión critica del "conectivismo". Universidad de Alcalá, 1 - 49.

\section{Conflicto de intereses / Competing interests:}

El autor declara que no incurre en conflictos de intereses.

Rol de los autores / Authors Roles:

No aplica.

Fuentes de financiamiento / Funding:

El autor declara que no recibió un fondo específico para esta investigación.

Aspectos éticos / legales; Ethics / legals:

El autor declara no haber incurrido en aspectos antiéticos, ni haber omitido aspectos legales en la realización de la investigación. 